\title{
Simulação Fenomenológica da Emissão de Material Particulado a Partir de uma Chaminé Industrial
}

\author{
F. M. Avelar ${ }^{1}$, M. B. Quadri ${ }^{1}$ \\ ${ }^{1}$ Departamento de Engenharia Química e Engenharia de Alimentos, Centro Tecnológico, \\ Universidade Federal de Santa Catarina, Florianópolis, Brasil. \\ Email: felipe.msla@gmail.com, mbq1959@hotmail.com
}

RESUMO - Trata-se da modelagem fenomenológica de uma chaminé expelindo partículas de vidro (ballotinis) com diâmetro médio $105 \mu \mathrm{m}$ utilizando dados do trabalho de Hall, que trata da dispersão entre outras desse tipo de partículas em túnel de vento. Com este modelo, uma vez validado, estuda-se o comportamento do material particulado em meio ao escoamento do ar ambiente, determinando-se o grau de dispersão e as distâncias percorridas pelas partículas para posteriormente verificar o risco de doenças respiratórias para a população próxima a uma indústria que emita material com diâmetro de $10 \mu \mathrm{m}$. Um modelo de escoamento laminar isotérmico foi inicialmente construído e posteriormente melhorado segundo um modelo isotérmico misto (laminar no exterior da chaminé e turbulento em seu interior). Testes de malha e de condições de contorno foram executados. $\mathrm{O}$ modelo desenvolvido apresentou resultados adequados, conforme os dados encontrados no trabalho supracitado.

Palavras chave: Material particulado, doenças respiratórias, simulação, fluidodinâmica, usina de açúcar, dispersão atmosférica.

\section{INTRODUÇÃO}

$\mathrm{O}$ atual cenário urbano e industrial tem causado em todo o mundo um aumento na quantidade de poluentes atmosféricos emitidos. O aumento desses poluentes, a absorção pelos humanos, bem como sua deposição no solo e em alimentos de uma forma geral, tem causado danos à saúde do homem.

O interesse econômico e social gera a necessidade da remoção ou diminuição dessas partículas do meio ambiente. A parte econômica é gerada através da indústria que se vê com a possibilidade de diminuir suas emissões através de equipamentos de retenção ou até um possível reuso no processo industrial. Já a parte social está ligada aos efeitos que este particulado causa a saúde da população (Cordeiro, 2010).As partículas ou particulados (pó) estão entre os poluentes que apresentam maiores riscos diretamente ao homem. Elas atacam os pulmões, aumentam as taxas de reação na atmosfera, reduzem a visibilidade e alteram os níveis de radiação solar que atingem o solo (De Carvalho Souza Jr., 2003).

Lora (2002) define material particulado (MP) como qualquer substância, à exceção da água 
pura, que existe como líquido ou sólido na atmosfera e que tem dimensões microscópicas ou sub-microscópicas, porém maiores que as dimensões moleculares. Devido ao seu pequeno diâmetro, o material particulado $\left(\mathrm{MP}_{10}\right)$ pode atingir as vias aéreas internas. Sendo assim, várias doenças podem ser identificadas tendo o material particulado como agente causador. Dentre elas podemos citar a Silicose e a Siderose que são causadas por inalação de pó de Sílica e Ferro respectivamente (De Almeida Filho, 2010).

O objetivo desta simulação, obtida através do programa de simulação COMSOL Multiphysics ${ }^{\circledR} 4.3 a$., é verificar se o modelo proposto consegue validar com precisão os resultados obtidos por Hall et al. (1998), que utiliza um túnel de vento experimental para mostrar o comportamento de partículas de vidro ao longo da distância percorrida. Um modelo fluidodinâmico e um modelo de partículas serão utilizados para se conhecer o comportamento dessa nuvem de partículas de vidro.

\section{MATERIAIS E MÉTODOS}

A simulação consiste de uma chaminé com uma exaustão de partículas de vidro em um meio com uma corrente de vento unidirecional, lembrando um túnel de vento. Os dados necessários para a simulação foram adquiridos junto ao trabalho de Hall et al. (1998). Um modelo fluidodinâmico não isotérmico foi simulado e em conjunto com estes resultado o modelo traçador de partículas foi colocado em prática para contagem, verificação e comparação de resultados.

\subsection{Modelo de Escoamento Laminar}

Para o modelagem foi utilizado um modelo de escoamento laminar e outro modelo de dispersão de partículas. Posteriormente, um modelo de escoamento turbulento foi adicionado a chaminé. O modelo de escoamento laminar é baseado no movimento de fluidos através da equação de Navier-Stokes, que apresenta solução para o campo de velocidade e pressão. Sua principal característica acaba sendo a possibilidade de incluir o material fluido e suas respectivas propriedades. O material selecionado é o ar e com ele as propriedades viscosidade dinâmica, densidade, coeficiente de transferência térmica. As equações gerais utilizadas são:

$$
\begin{aligned}
& \frac{\partial p}{\partial t}+\nabla \cdot(\rho \mathbf{u})=0 \\
& \rho \frac{\partial u}{\partial t}+\rho(\mathbf{u} \cdot \nabla) \mathbf{u}=\nabla \cdot[-p \mathbf{I}+\tau]+\mathbf{F} \\
& \rho C_{p}\left(\frac{\partial \mathrm{T}}{\partial t}+(\mathbf{u} \cdot \nabla) \mathrm{T}\right)=-(\nabla \cdot \mathbf{q})+\mathbf{\tau}: \mathbf{S}-\frac{T}{\rho} \frac{\partial \rho}{\partial T} \mathrm{I}_{\rho}\left(\frac{\partial p}{\partial t}+(\mathbf{u} \cdot \nabla) p\right)+\mathbf{Q}
\end{aligned}
$$

Onde $\mathbf{s}=\frac{1}{2}\left(\nabla \mathbf{u}+(\nabla \mathbf{u})^{T}\right)$ 


\subsection{Modelo de Escoamento Turbulento}

Nas partes internas do bico e da chaminé, foi utilizado o modelo turbulento para modelar o seu comportamento já que sua velocidade atinge valores muito altos para um regime laminar. Foi utilizado o modelo k- $\varepsilon$ já que este é um dos mais utilizados para aplicações industriais. Isto introduz duas equações adicionais de transporte e duas variáveis dependentes: a energia cinética turbulenta, $k$, e da taxa de dissipação de energia turbulência, $\varepsilon$ :

$\rho \frac{\partial k}{\partial t}+\rho(\mathbf{u} \cdot \nabla) k=\nabla \cdot\left[\left(\mu+\frac{\mu_{T}}{\sigma_{k}}\right) \nabla k\right]+P_{k}-\rho \epsilon$

$P_{k}=\mu_{T}\left(\nabla \mathbf{u}:\left(\nabla \mathbf{u}+(\nabla \mathbf{u})^{\mathrm{T}}\right)-\frac{2}{3}(\nabla \cdot \mathbf{u})^{2}\right)-\frac{2}{3} \rho \mathrm{k} \nabla \cdot \mathbf{u}$

$\rho \frac{\partial \varepsilon}{\partial t}+\rho \mathbf{u} \cdot \nabla \varepsilon=\nabla \cdot\left(\left(\mu+\frac{\mu_{T}}{\sigma_{\varepsilon}}\right) \nabla \varepsilon\right)+C_{\varepsilon 1} \frac{\varepsilon}{k} P_{k}-C_{\varepsilon 2} \rho \frac{\varepsilon^{2}}{k}$

$\mu_{T}=\rho C_{\mu} \frac{\mathbf{k}^{2}}{g}$

\subsection{Modelo de Dispersão de Partículas}

O modelo traçador de partículas fornece uma descrição Lagrangiana de um problema de resolução de equações diferenciais ordinárias usando a lei de Newton do movimento. Esta lei de Newton requer especificação da massa das partículas e todas as forças que atuam sobre a partícula. As forças que atuam sobre as partículas podem ser divididas em duas categorias; aquelas devido a campos externos e, devido a interações entre as partículas.

$$
\frac{d\left(m_{p} v\right)}{d t}=F_{t}
$$

Para cada partícula, uma equação diferencial é resolvida para cada um de seus vetores componentes de posição. Isto significa que as três equações diferenciais ordinárias são resolvidas para cada partícula em 3D. Em cada intervalo de tempo, as forças que atuam sobre cada partícula são consultadas a partir dos campos externos na atual posição de partícula. Se as forças de interação partícula-partícula são incluídas no modelo, em seguida, são adicionados à força total. A posição da partícula é então atualizada, e o processo se repete até que o tempo do fim especificado para a simulação é atingido. As partículas utilizadas neste modelo são do mesmo tamanho e material utilizado por Hall et al. (1998) em seu trabalho, partículas de vidro com $105 \mu \mathrm{m}$. 
As seguintes simplificações foram consideradas:

- O modelo 3-D construído pela metade utilizando-se a condição de simetria como meio de economizar malha e aliviar a modelagem com dimensões de 300 m x 100 m x 150 m;

- Regime laminar (fora da chaminé) e regime turbulento (na chaminé e no tubo de injeção) com equações Reynolds averaged Navier-Stokes (RANS) e um modelo de turbulência k$\varepsilon$

- O material selecionado é o ar seco e contém as propriedades viscosidade dinâmica, massa específica, calor especifico, condutibilidade elétrica, coeficiente de transferência térmica.

- Foi utilizado discretização do tipo P1 + P1;

- Fluido compressível;

- Para força de arraste a lei de arraste de Schiller-Naumann foi aplicada ao modelo de partículas;

- Propriedades das partículas newtonianas;

- O modelo foi construído ao longo da direção x, direção na qual se aplica o escoamento.

- Existe uma força gravitacional na direção -z;

- Existem coletores no chão (B5) para fazer a contagem das partículas que caíram comparando assim com artigo proposto por Hall et al. (1998).

- As partículas são de vidro e tem o diâmetro médio de $105 \mu \mathrm{m}$.

- Velocidade de injeção de partículas de $110 \mathrm{~m} / \mathrm{s}$ e velocidade do vento de $7 \mathrm{~m} / \mathrm{s}$.

\subsection{Condições de Contorno Para Modelo de Escoamento}

As condições iniciais e de contorno consideradas no modelo estão apresentadas nas Figuras 1 e 2 .

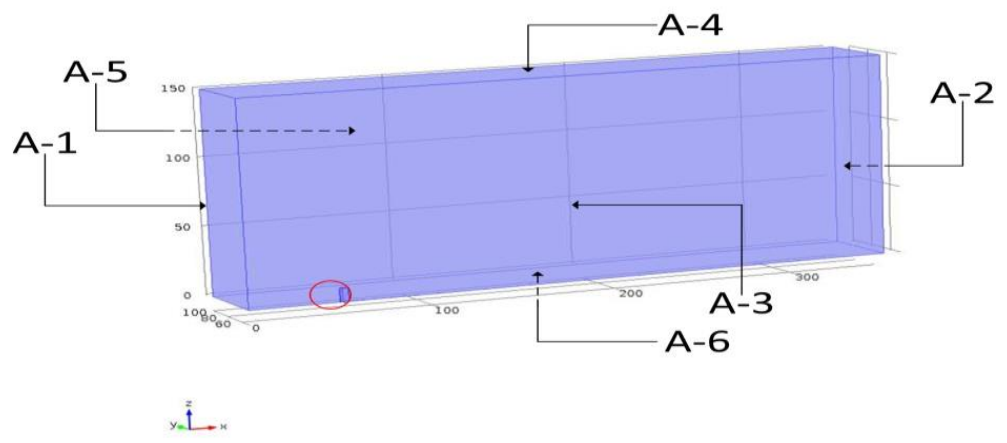

Figura 1 - Indicação das condições de contorno no domínio onde se insere a chaminé

A1, C1 - Inlet, $\mathbf{u}=-\mathrm{U}_{0} \mathbf{n}$ 
A2, A4, A5 - Outlet, for $p=p_{0},\left[\mu\left(\nabla \mathbf{u}+(\nabla \mathbf{u})^{T}\right)-\frac{2}{3} \mu(\nabla \times \mathbf{u}) \mathbf{I}\right] \times \mathbf{n}=0$;

A3, B4 - Symmetry, $\mathbf{u} \times \mathbf{n}=\mathbf{0},\left(-\rho \mathbf{I}+\left(\mu\left(\nabla \mathbf{u}+(\nabla \mathbf{u})^{T}\right)-\frac{2}{3} \mu(\nabla \times \mathbf{u}) \mathbf{I}\right)\right) \mathbf{n}=0$;

A6, B1, B2, B3, C2, C3 - No slip, $\mathbf{u}=0$

Uma condição inicial de velocidade foi assumida no sistema de forma a facilitar a convergência das simulações.

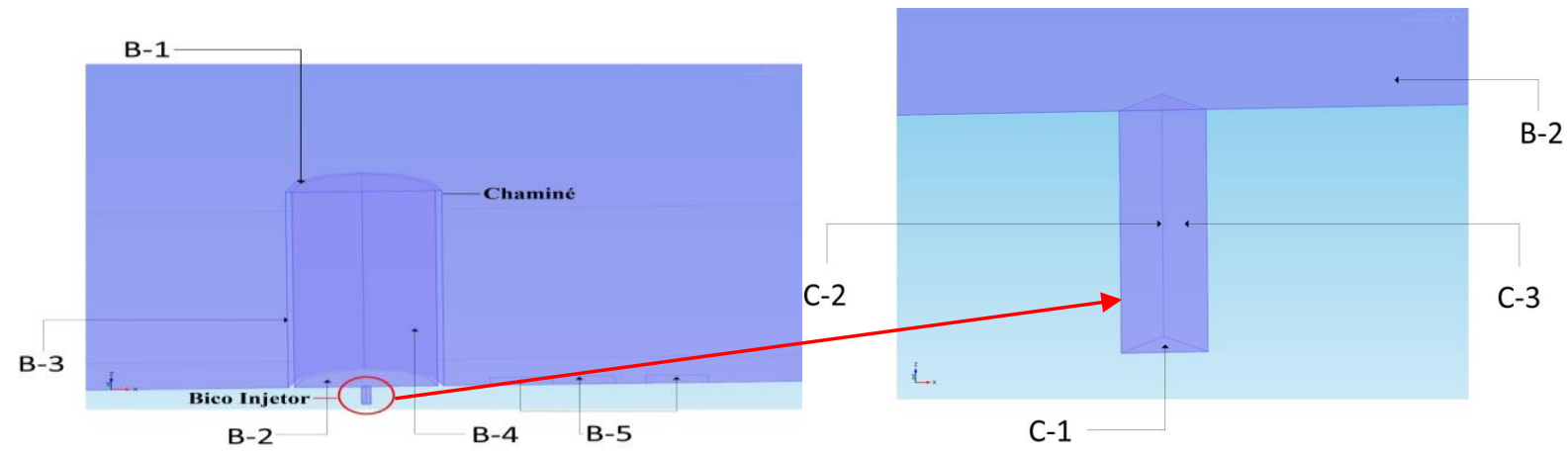

Figura 2- Indicação na chaminé e injetor das condições de contorno assumidas no modelo

\subsection{Condições Assumidas Para o Modelo de Dispersão de Partículas:}

A6, B2, B3 - Freeze, $\mathbf{v}=\mathbf{v}_{\mathrm{c}}$

$\mathrm{C} 1$ - Inlet -10 mil partículas, $\mathbf{v}=\mathbf{v}_{0}$ e $\mathbf{q}_{p}=\mathbf{q}_{0}$

B1, B4, C2, C3 - Bounce, $\mathbf{v}=\mathbf{v}_{\mathrm{c}}-2\left(\mathbf{n} \times \mathbf{v}_{\mathrm{c}}\right) \mathbf{n}$

A1, A2, A4, A5 - Outlet - Equação (13).

A3 - Stick, $\mathbf{v}=0$

Onde $\mathbf{v}_{\mathbf{c}}$ é a velocidade da partícula quando atinge a parede.

\section{PROCEDIMENTO NUMÉRICO}

Basicamente, a solução aproximada para o modelo é feita em dois passos. Primeiro, o perfil de velocidade é calculado. Em seguida, utilizando este perfil, o modelo de dispersão de partículas é obtido. O perfil de velocidade é calculado utilizando o método de elementos finitos 
que consiste em descrever o modelo com um número finito de parâmetros ou graus de liberdade.

\subsection{Malha}

Após inúmeros testes com malhas, uma malha preferencial foi definida. Esta foi escolhida levando em conta a capacidade desta em apresentar comportamento semelhante a da malha mais refinada com um gasto de tempo de simulação mais próximo do tempo gasto por uma malha mais grosseira. Esta malha preferencial é calibrada para fluidodinâmica e ainda sofre alguns ajustes para o seu refinamento. Pelo fato do domínio ser de grandes dimensões e apresentar subdomínios de diferentes ordens de grandeza, foram necessárias malhas de transição nas fronteiras desses subdomínios de modo a se obter a convergência das soluções.

\section{RESULTADOS E DISCUSSÕES}

O modelo de emissão de ballotinis foi feito para ser comparado com o trabalho "Deposition of large particles from warehouse fire plumes-a small-scale wind tunnel model study" de Hall et al. (1998) que baseia-se em um túnel de vento com uma condição de velocidade de injeção de partículas no meio, condição S2.

Desde os primeiros ensaios de simulação, as condições de contorno foram testadas para que o programa aplicasse exatamente o que estava sendo propondo e executasse as simulações do modo que a convergência do método numérico atingisse os valores mais baixos possíveis. Isto só foi possível graças à extensa lista de condições de contorno para fluxo laminar e turbulento.

Uma nova malha para a validação foi construída de forma a se obter resultados mais precisos e coerentes com os fenômenos que ocorrem no interior da chaminé. Assim, promoveuse um refinamento axial no interior da chaminé de forma a melhor representar o escoamento nessa região e minimizar o efeito de deformação do campo de velocidades junto à saída da mesma, como mostra a Figura 3. Observa-se o escoamento visto com linhas de corrente e sem linhas de corrente, notando-se uma suavização ao longo do perfil dentro da chaminé com o reforço feito na malha daquela região. Também é possível observar uma zona de baixas velocidades à sombra do escoamento do lado de fora da chaminé.

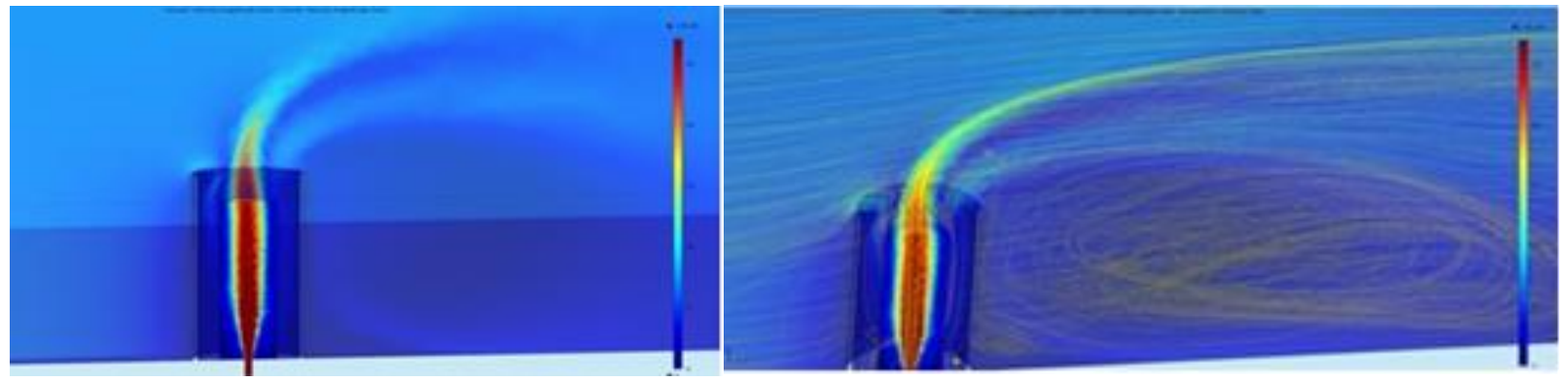

Figura 3 - Vista do campo de velocidades na chaminé e à sombra do escoamento obtido com a nova malha para o modelo de validação visto com e sem linhas de corrente 
O trabalho de Hall et al. (1998) considera uma concentração adimensional K para medir a massa de partículas que cai em seus coletores. A equação (18) mostra como é calculado o adimensional $\mathrm{K}$ :

$$
\mathrm{K}=\frac{\mathrm{m}}{\mathrm{M}} \cdot \frac{\mathrm{UH}^{2}}{\mathrm{v}_{\mathrm{f}}}
$$

Os valores de $\mathrm{m}$ foram encontrados a partir das contagens dos depósitos de partículas nos coletores. Os valores de $\mathrm{M}$ foram conhecidos diretamente pelo número de injeção de partículas. Os valores de vf eram conhecidos a partir das características das partículas.

Alguns testes foram efetuados para o aperfeiçoamento do modelo. Um desses aperfeiçoamentos seria a condição de contorno no tubo injetor para o modelo de escoamento. $\mathrm{O}$ modelo sem escorregamento (No Slip) tem como consequência um melhor espalhamento das partículas no modelo de dispersão de partículas como pode se ver na Figura 4. O modelo de escoamento com condição No Slip produziu melhores resultados ao se comparar com aqueles apresentados neste trabalho utilizado para validação. Esta condição foi então escolhida para ser aplicada no restante das simulações.
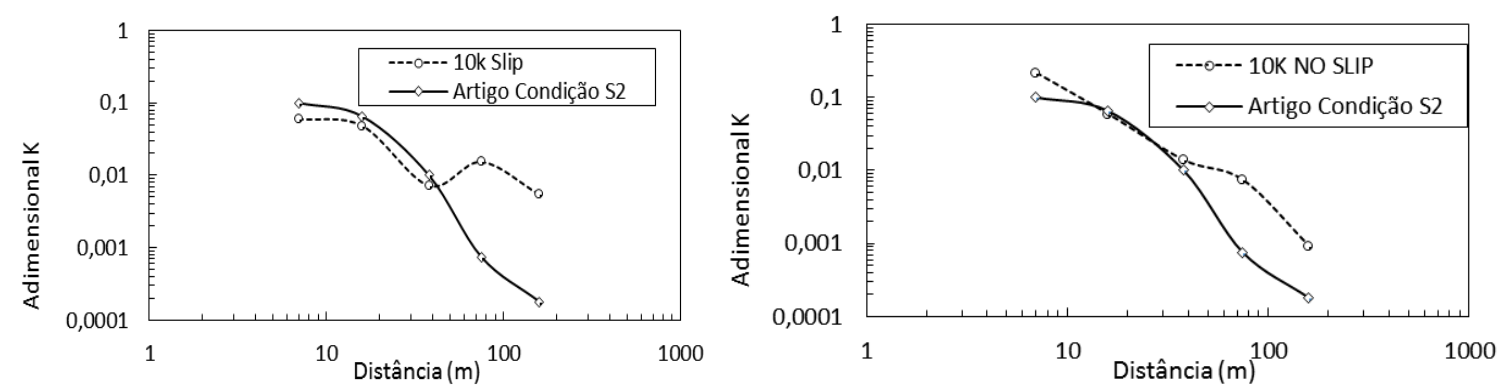

Figura 4 - Gráficos comparando o resultado de uma condição de contorno Slip (a) e No Slip (b) e com o resultado de Hall et al. (1998) na condição S2

Além da quantidade de elementos na malha, outro fator que determinou o tempo de cálculo gasto em cada simulação foi a condição de contorno nas paredes da chaminé e a presença da parede de simetria. Com a condição Freeze as partículas simplesmente paravam ao se chocarem com essas paredes e assim diminuía-se o número das mesmas na contagem final de partículas nos coletores. Com a nova condição Bounce as partículas não mais paravam ao se chocarem com as paredes, mas sim eram rebatidas elasticamente, conservando-se o seu momentum. Esta condição reduz o tempo gasto com a simulação em aproximadamente $80 \%$.

A condição de parede Bounce resulta em números mais expressivos do adimensional $\mathrm{K}$. Isso pode ser notado na Figura 5. Pelo fato das partículas não ficarem mais retidas nas paredes da chaminé, o número de partículas que caem nos coletores é maior.

Com esta nova condição de contorno, dois novos modelos foram testados. O primeiro considerou a opção Bounce apenas na chaminé e na parede de simetria adotou-se a condição 
Freeze. Um comportamento similar ao observado no modelo anterior com a condição No Slip foi encontrado, constando-se uma diferença de $25 \%$ a mais no número de partículas encontradas nos coletores para a opção Bounce.
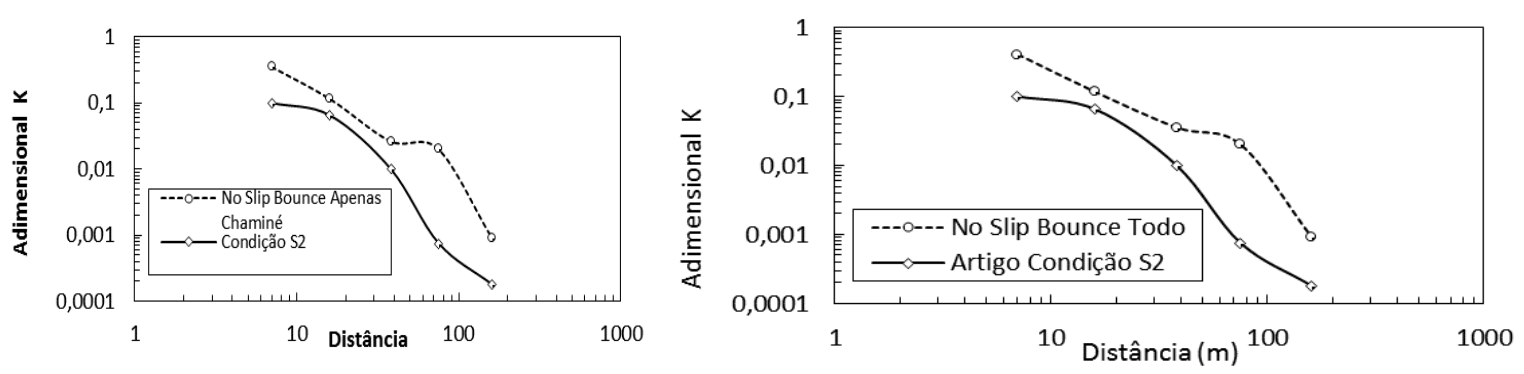

Figura 5 - Gráfico comparando o resultado de uma condição de contorno No Slip / Bounce (apenas na chaminé) (a) e No Slip / Bounce (chaminé + condição de simetria) (b) com o resultado de Hall et al. (1998) na condição S2

O segundo modelo considerou a condição de contorno Bounce tanto na chaminé quanto na parede de simetria, a quantidade de partículas encontradas nos coletores foi ligeiramente maior do que no modelo anterior, com Bounce apenas na chaminé, porém o mesmo comportamento foi observado.

Para uma avaliação das malhas entre si, apresentam-se os gráficos da Figura 6. Neles consegue-se verificar um desvio padrão máximo de 4,5\% de diferença entre as malhas. Com este resultado se tem respaldo necessário para utilizar uma malha mais leve e consequentemente exigir menos processamento computacional.
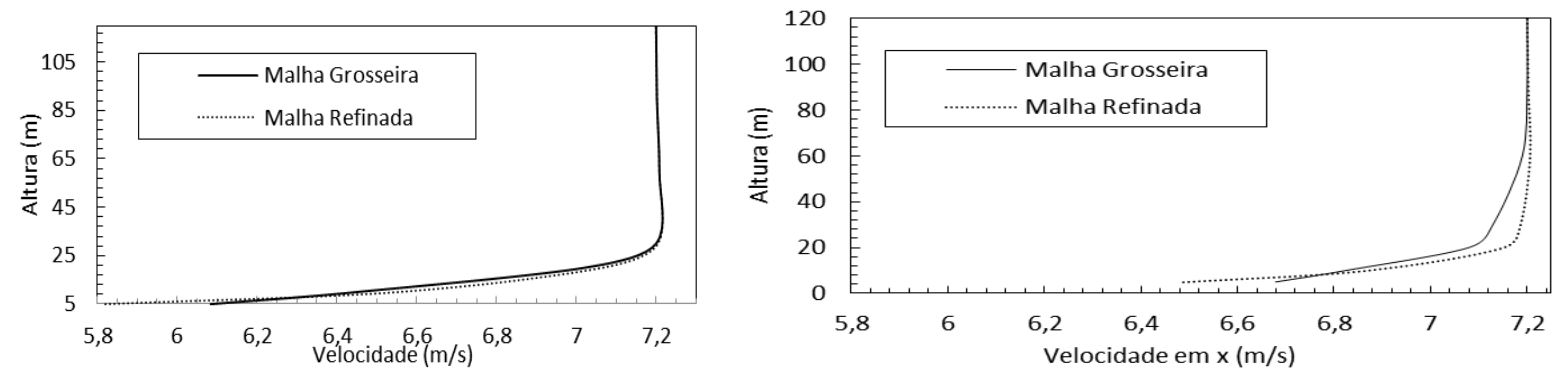

Figura 6 - Gráficos de avaliação das duas malhas utilizadas para o modelo de validação a 50 m (a) e $275 \mathrm{~m}$ (b) da chaminé

\section{CONCLUSÃO}

A abordagem por meio da técnica dos elementos finitos do problema da emissão de material particulado, a partir de uma chaminé em sistema não-isotérmico, mostrou-se uma poderosa ferramenta para o estudo da fluidodinâmica e da trajetória das partículas sob condições operacionais conhecidas. 
O modelo ao considerar a emissão de partículas do tipo ballotinis reproduziu adequadamente o comportamento experimental obtido de um trabalho de referência, que estudou o fenômeno de dispersão de partículas de vidro a partir de uma fonte localizada no interior de um túnel de vento. A comparação entre o modelo proposto e o artigo de túnel de vento indica que o modelo é adequado para prever a dispersão das partículas.

\section{NOMENCLATURA}

$C_{p}-$ Calor específico a pressão constante $(\mathrm{J} /(\mathrm{kgK}))$

$\mathbf{F}$ - Vetor força $\left(\mathrm{N} / \mathrm{m}^{3}\right)$

$F_{t}-$ Força exercida na partícula $(\mathrm{N})$

$\mathrm{H}$ - Altura da chaminé (m)

I - Matriz identidade

$\mathrm{K}$ - Adimensional de concentração

$\mathrm{m}$ - Massa nos coletores $(\mathrm{kg})$

$\mathrm{M}$ - Massa total injetada $(\mathrm{kg})$

$m_{p}$ - Massa da partícula $(\mathrm{kg})$

n - Limite normal exterior $\left(\mathrm{kg} /\left(\mathrm{m}^{2} / \mathrm{s}\right)\right)$

$p$ - Pressão $(\mathrm{Pa})$

$\mathrm{p}_{\mathrm{A}}$ - Pressão do componente A $(\mathrm{Pa})$

$\mathrm{p}_{0}-$ Pressão de entrada $(\mathrm{Pa})$

$\mathrm{p}_{\infty}$ - Pressão de saída $(\mathrm{Pa})$

$\mathbf{q}$ - Fluxo de calor por condução $\left(\mathrm{W} / \mathrm{m}^{2}\right)$

$\mathbf{q}_{\mathbf{p}}$ - Posição da partícula $\mathbf{q}_{\mathbf{0}}$ - Posição inicial da partícula

$q_{0}$ - Fluxo de calor para o interior $\left(\mathrm{W} / \mathrm{m}^{2}\right)$

$\mathbf{S}$ - Tensor de deformação (1/s)

$T$ - Matriz transposta

$\mathrm{T}$ - Temperatura absoluta $(\mathrm{K})$

$\mathrm{t}$ - Tempo (s)

$\mathrm{U}$ - Campo de valocidade

$\mathbf{u}$ - Vetor de velocidade do fluido $(\mathrm{m} / \mathrm{s})$

$v$ - Velocidade da partícula $(\mathrm{m} / \mathrm{s})$

$\mathbf{v}$ - Vetor de velocidade da partícula $(\mathrm{m} / \mathrm{s})$

$\mathbf{v}_{\mathbf{c}}-$ Velocidade de contato $(\mathrm{m} / \mathrm{s})$

$\mathrm{v}_{\mathrm{f}}-$ Velocidade terminal das partículas

$(\mathrm{m} / \mathrm{s})$

$\mathbf{v}_{\mathbf{0}}$ - Velocidade inicial da partícula $(\mathrm{m} / \mathrm{s})$

$\mu$ - Viscosidade dinâmica (Pa.s)

$\rho$ - Densidade $\left(\mathrm{kg} / \mathrm{m}^{3}\right)$

$\tau-$ Tensor de estresse viscoso $(\mathrm{Pa})$

\section{REFERÊNCIAS}

CORDEIRO, G. C.; TOLEDO FILHO, R. D.; FAIRBAIRN, E.M.R. "Caracterização de cinza do bagaço de cana-de-açúcar para emprego como pozolana em materiais cimentícios”. Química Nova,. v. 32, n. 1, p. 82-86, 2009.

DE ALMEIDA FILHO, F. (2010), "Monitoramento e controle de emissão de material particulado em uma fonte estacionária."

DE CARVALHO SOUZA JR, J. A. (2003), Emissões em processos de combustão, Livraria UNESP.

HALL, D. J., V. KUKADIA, et al. (1998), "Deposition of large particles from warehouse fire plumes - a small-scale wind tunnel model study." Journal of hazardous materials 59(1): 13-29.

LORA, E. E. S., Prevenção e controle da poluição nos setores energético, industrial e de transporte. Interciência, 2002.

RQARMC (2012), Relatórios de qualidade do ar de Curitiba e Região Metropolitana. Em: <http://www.iap.pr.gov.br/modules/conteudo/conteudo.php?conteudo=131> Acesso 25/01/2012. 